\section{PCOS: Matter of Eggs and Far Beyond}

Pooja Vaswani*

Department of Obstetrics and Gynecology, Universal Hospital, Abu Dhabi, United Arab Emirates

\begin{abstract}
Polycystic Ovary Syndrome (PCOS) is one of the most common endocrinological disorder among women of reproductive age. It is a heterogeneous disorder and most women present to the clinician with menstrual irregularities, sub fertility, hirsutism and acne. Many women present at a younger age when age related medical conditions are generally not prevalent. While the woman's reproductive and cosmetic concerns are definitely important but as clinicians our role is not restricted just to these concerns but to look beyond and evaluate a woman's risk for development of metabolic diseases and long term implications of PCOS. The aim of this article is to provide awareness about important metabolic disorders associated with PCOS.

It is important to treat women with PCOS with a holistic approach keeping in mind that contrary to what the name implies, PCOS implications are not restricted just to sex organs but go way beyond affecting many other organs in the body which can have profound effect on long term health of the women.
\end{abstract}

\section{Introduction}

Quite many times in our daily practice we come across women who are diagnosed to have Polycystic Ovarian Syndrome (PCOS), the incidence of which is on an increase. It affects about $10 \%$ of women [1]. It's the word "PCOS" that catches any woman's attention, as more often than not she would have heard about PCOS from her friends/ mother or sisters. Immediate question that comes to the mind of the woman is "will I be able to conceive?" Maternal instinct is paramount for any women.

While myths remain persistent in women with PCOS that they may not get pregnant, the reality is that the infertility associated with PCOS is highly treatable leading to pregnancy in most women with PCOS $[2,3]$. The good news is that many women with PCOS will have increase in fertility just through lifestyle changes and modest weight loss $[2,3]$. In other women pregnancy can be achieved successfully

*Corresponding author: Pooja Vaswani, Department of Obstetrics and Gynecology, Universal Hospital, Abu Dhabi, United Arab Emirates, Tel: +971 552029740; E-mail: poojarvaswani@yahoo.co.in

Citation: Vaswani P (2017) PCOS: Matter Of Eggs And Far Beyond. J Hum Endocrinol 2: 006.

Received: April 10, 2017; Accepted: May 10, 2017; Published: May 24, 2017

Copyright: (C 2017 Vaswani $\mathrm{P}$, This is an open-access article distributed under the terms of the Creative Commons Attribution License, which permits unrestricted use, distribution, and reproduction in any medium, provided the original author and source are credited. with basic infertility treatments. There will remain some women with PCOS who need additional help in conceiving which is in the form of In Vitro Fertilization (IVF) which is a highly effective form of treatment of infertility in women with PCOS [3].

Most women with PCOS often think losing weight is an almost impossible task and they question "Can diet and exercise help me conceive?" The benefits of weight reduction are much more than it seems. Indeed just by losing 5-10 percent of body weight, a woman can reduce central fat by up to $30 \%$ thus improving insulin sensitivity and restoring ovulation [3]. Weight loss results in improved ovulatory function, improved chances of conception, a safer pregnancy for both the mother and baby, and if the woman needs, a better response to fertility medications $[4,5]$. Weight loss has also been shown to reduce other symptoms such as excessive and abnormal hair growth, acne, and balding [3-6].

Many women with PCOS feel that the reason for not ovulating is because their ovaries contain fewer eggs than normal. However the fact is women with PCOS may actually be endowed with a larger ovarian reserve at birth $[7,8]$. The increased density of pre-antral follicles can be explained by a higher population of primordial follicles. Possible mechanisms for which are, more primordial germ cells entering the ovary during fetal life, more cell divisions of germ cells or granulose cells which can lead to more follicles being formed. Another explanation for the larger stock of small pre-antral follicles and longevity of this stock is due to less follicle loss by atresia in polycystic ovaries $[7,8]$. However despite an increased stock, many women with PCOS have anovulatory cycles owing to several hormonal and other factors that prevent a few healthy follicles from maturation and ovulation $[3,7,8]$. There is an abnormal endocrine environment that includes hypersecretion of luteinizing hormone, insulin and androgens which leads to suppression of FSH leading to inhibition of maturation of otherwise healthy follicles [7]. There is emerging evidence that there may be an intrinsic abnormality of folliculogenesis in PCOS that affects very early stages of follicle development that are gonadotrophin independent [7]. There is also evidence that abnormal signaling of anti-mullerian hormone that affects local follicle to follicle signals may have a role in disordered folliculogenesis [7]. Apart from these, other local regulators like insulin-like growth factors and sex steroids have been implicated in disordered follicular development and thus leading to anovulation [7].

Primary cause of many symptoms in majority of women with PCOS is "insulin resistance" [1-3,9] which simply means different cells in the body are either "resistant" to or are "under-responding" to the hormone insulin. Therefore, the cells do not get appropriate "signals" to perform a multitude of many tasks. In the absence of coordinated signals the entire body goes through a roller coaster of hormonal imbalance and distress, leading to an excess of male hormones which ultimately leads to many of symptoms of PCOS like weight gain, central obesity, skin and hair problems $[4,5]$. It is well known that obesity is observed in approximately $60 \%$ of women with PCOS [1]. The hyperinsulinaemia owing to insulin resistance promotes further weight gain creating a vicious cycle [9-11]. Apart from hyperinsulinaemia, a new hypothesis suggests that Follicle Stimulating Hormone (FSH) activity may be somehow blocked at the ovarian level owing to defects in primary cellular control mechanisms which result in chronic 
anovulation and hyperandrogenism [12]. There is also a high prevalence of PCOS in families and this familial clustering suggests a genetic origin of the disorder [13]. Most recent work shows that there may be a strong cause and effect relationship between Aquaporins 7, 8 and 9 (AQPs) expression in (atleast) adipocytes and granulose cells which affects follicle growth and quality. Aquaporins are transmembrane proteins which are able to transport water molecules (and in some cases also small uncharged molecules as glycerol) through biological membranes. Upcoming research suggests a possible novel approach to treatment, especially in the case of diseases leading to systemic effects as aquaporins are regarded as potential drug targets [14].There is also some evidence that Bisphenol A which is found in environment may have a role in pathophysiology of PCOS affecting endocrine and/or metabolic functions thus indicating possible role of occupational hazard in PCOS pathophysiology [15].

As gynecologists we meet women with PCOS whose usual concerns are either cosmetic or reproductive. However, if we have far sight and look beyond, there are more serious concerns which are likely to happen beyond the reproductive age. Many studies without doubt confirm that the insulin resistance associated with PCOS can expose the women to higher risk of metabolic syndrome including hypertension; hyperlipidemia, diabetes and cardiovascular disease $[9,10]$. It is reported that more than $20 \%$ of obese women with PCOS will have impaired glucose tolerance after the age of 30 [16,17]. By the age of 40 , almost $40 \%$ women with PCOS develop pre-diabetes or diabetes. In other words, prevalence of type 2 diabetes in women with PCOS is 7 times higher than controls $[17,18]$. However it is important to remember that the risk of developing type 2 diabetes is also increased in non-obese women with PCOS. Thus PCOS seems to be an independent risk factor for development of type 2 diabetes in middle age $[19,20]$.

The increased in cardiovascular risk in PCOS women is owing to two mechanisms, direct atherogenic action due to insulin resistance and the adverse effect of the lipoprotein profile seen in PCOS [21]. The risk of heart attack is also increased four to seven times in these women [22]. It appears that in PCOS women risk factors for development of atherosclerotic conditions, hypertension and myocardial infarction are present at an earlier age than in women without PCOS [22]. Obstructive Sleep Apnea (OSA) and daytime somnolence are also found to be three times more common in PCOS patients [9].

Worldwide survey of healthcare professionals has led to suggestion that the name PCOS is confusing and can be misleading as it indicates involvement only of ovaries which is far from reality. Instead "Metabolic reproductive syndrome" has been suggested as the new name for PCOS keeping in mind the long term metabolic risks associated with PCOS involving many other organs apart from ovaries [23-25].

Apart from the above metabolic disorders, women with PCOS are also at an increased risk of developing uterine, ovarian and possibly also breast cancer in later life [9]. Chronic anovulation predisposes women to endometrial cancer and this risk is reported to be three times in PCOS women as compared to women without PCOS [9]. These women thus should be evaluated for these conditions accordingly on a more frequent basis than non-PCOS women.

Keeping in mind long term implications of PCOS, there is a need for a holistic approach to the disease process. Every responsible gynecologist should utilize every potential opportunity to evaluate and educate the patients about ways to prevent these potentially threatening long term metabolic disorders by intervening at the earliest. A long term follow up beyond reproductive age to prevent the co-morbidities and yearly blood screening for hyperlipidemia and diabetes is recommended [26].

\section{Conclusion}

Polycystic ovarian syndrome that was once considered to be purely related to sex organs should no longer be considered a purely gynecological disorder. There needs to be widespread awareness about metabolic complications of PCOS and education that "sex hormones" do not affect only the reproductive organs but many other organs in the body leading to potentially serious long term complications.

\section{References}

1. Daniilidis A, Dinas K (2009) Long term health consequences of polycystic ovarian syndrome: a review analysis. Hippokratia 13: 90-92.

2. Carmina $E$ (2006) Metabolic syndrome in polycystic ovary syndrome. Minerva Ginecol 58: 109-114.

3. Balen AH (2007) Managing anovulatory infertility and polycystic ovary syndrome. BMJ 335: 663-666.

4. Barber TM, Wass JA, McCarthy MI, Franks S (2007) Metabolic characteristics of women with polycystic ovaries and oligo-amenorrhoea but normal androgen levels: implications for the management of polycystic ovary syndrome. Clin Endocrinol 66: 513-517.

5. Chavez-Ross A, Franks S, Mason HD, Hardy K, Stark J, et al. (1997) Modelling the control of ovulation and polycystic ovary syndrome. J Math Biol 36: 95-118.

6. Clark AM, Ledger W, Galletly C, Tomlinson L, Blaney F, et al. (1995) Weight loss results in significant improvement in pregnancy and ovulation rates in anovulatory obese women. Hum Reprod 10: 2705-2712.

7. Franks S, Stark J, Hardy K (2008) Follicle dynamics and anovulation in polycystic ovary syndrome. Hum Reprod Update 14: 367-378.

8. Webber LJ, Stubbs S, Stark J, Trew GH, Margara R, et al. (2003) Formation and early development of follicles in the polycystic ovary. Lancet 362: 10171021.

9. Shah D, Rasool S (2015) PCOS and Metabolic Syndrome: The Worrisome Twosome? Endocrinol Metab Synd 4: 169.

10. Moini A, Javanmard F, Eslami B, Aletaha N (2012) Prevalence of metabolic syndrome in polycystic ovarian syndrome women in a hospital in Tehran. Iran J Reprod Med 10: 127-130.

11. Ali AT (2015) Polycystic ovary syndrome and metabolic syndrome. Ceska Gynekol 80: 279-289.

12. Matalliotakis I, Kourtis A, Koukoura O, Panidis D (2006) Polycystic ovary syndrome: etiology and pathogenesis. Arch Gynecol Obstet 274: 187-197.

13. ESHRE Capri Workshop Group (2008) Genetic aspects of female reproduction. Hum Reprod Update 14: 293-307.

14. Wawrzkiewicz-Jałowiecka A, Kowalczyk K, Pluta D, Blukacz L, Madej P (2017) The role of aquaporins in polycystic ovary syndrome-A way towards a novel drug target in PCOS. Medical Hypotheses 102: 23-27.

15. Vahedi M, Saeedi A, Poorbaghi SL, Sepehrimanesh M, Fattahi M (2016) Metabolic and endocrine effects of bisphenol A exposure in market seller women with polycystic ovary syndrome. Environ Sci Pollut Res Int 23: 23546-23550.

16. Sharma A,Yousef M (2000) Recent development in polycystic ovary syndrome. In: Studd J (ed.). Progress in Obstetrics and Gynecology. Churchill Livingstone, London, UK.

17. Wijeyaratne CN, Balen AH, Barth JH, Belchetz PE (2002) Clinical manifestations and Insulin Resistance (IR) in Polycystic Ovary Syndrome (PCOS) among South Asians and Caucasians: is there a difference? Clin Endocrinol 57: 343-350. 
18. Gopal M, Duntley S, Uhles M, Attarian H (2002) The role of obesity in the increased prevalence of obstructive sleep apnea syndrome in patients with polycystic ovarian syndrome. Sleep Med 3: 401-404.

19. Clinical green top guidelines (2007) RCOG.

20. Jedel E, Kowalski J, Stener-Victorin E (2008) Assessment of health-related quality of life: Swedish version of polycystic ovary syndrome questionnaire. Acta Obstet Gynecol Scand 87: 1329-1335.

21. Pierpoint T, McKeigue PM, Issacs AJ, Wild SH, Jacobs HS (1998) Mortality of women with polycystic ovary syndrome at long-term follow-up. J Clin Epidemiol 51: 581-586.

22. British Cardiac Society, British Hypertension Society, Diabetes UK, HEART UK, Primary Care Cardiovascular Society, Stroke Association (2005) JBS 2 Joint British Societies' guidelines on prevention of cardiovascular disease in clinical practice. Heart 91: 1-52.
23. The Rotterdam ESHRE/ASRM-Sponsored PCOS consensus workshop Group (2004) Revised 2003 consensus on diagnostic criteria and long term health Risks related to Polycystic Ovary Syndrome (PCOS). Human Reproduction 19: 41-47.

24. Gambineri A, Pelusi C, Vicennati V, Pagotto U, Pasquali R (2002) Obesity and the polycystic ovary syndrome. Int J Obes Relat Metab Disord 26: 883896.

25. Teede H (2016) 3-CE-SY17. Presented at: American Diabetes Association's Scientific Sessions, New Orleans, USA.

26. Hopkinson ZEC, Sattar N, Fleming R, Greer IA (1998) Polycystic ovarian syndrome: the metabolic syndrome comes to gynaecology. BMJ 317: 329-332. 